\title{
Contrôle de l'expression des gènes de différenciation adipocytaire : cycle cellulaire et hormones
}

\author{
P Grimaldi \\ CNRS, Centre de biochimie, laboratoire de biologie du développement du tissu adipeux, \\ UPR73, faculté des sciences, Parc Valrose, 06034 Nice, France
}

(15 réunion du groupe Développement, INRA, Paris, 24-26 mai 1989)

\begin{abstract}
Résumé - La différenciation adipocytaire se traduit par l'activation de nombreux gènes et est contrôlée par différents facteurs adipogéniques et mitogéniques. L'utilisation de modẻles cellulaires a conduit à la distinction de plusieurs étapes dans la conversion du préadipocyte en adipocyte, à la caractérisation des événements cellulaires et moléculaires impliqués dans ces étapes et à la description du mode d'action de diverses hormones adipogéniques. L'arrêt prolongé du préadipocyte dans la phase G1/S du cycle cellulaire provoque l'activation d'une série de gènes (pOb24, lipoprotéine lipase, etc.). L'expression des gènes de différenciation terminale, en particulier de ceux codant pour les enzymes de la lipogénèse, est plus tardive et nécessite d'une part le traitement des cellules par des hormones "permissives" (hormone somatotrope, triiodothyronine) et d'autre part des mitoses spécifiques aux cellules ayant déjà exprimé la première partie du programme de différenciation. L'insuline exerce dans les cellules différenciées, un effet modulateur sur l'expression des gènes de différenciation terminale. In vivo, une distinction entre événements précoces et tardifs, lors de la conversion adipocytaire, peut également être proposée. Une phase de prolifération initiale conduirait à la création d'un pool de préadipocytes exprimant uniquement les marqueurs précoces de différen. ciation (ARNm pOb24, lipoprotéine lipase). Certaines de ces cellules effectueraient des mitoses puis exprimeraient le programme de différenciation terminale; d'autres préadipocytes resteraient présents même à l'âge adulte, et pourraient entrer en phase de différenciation terminale sous l'influence de stimuli mitogéniques et adipogéniques non encore identifiés.
\end{abstract}

adipocyte / différenclation / cycle cellulaire / régulation hormonale

Summary - Expression of adipose differentiation-related genes: cell cycle and hormonal regulation. The adipose conversion of cultured preadipose cells involves the activation of numerous genes and is controlled by various adipogenic and mitogenic factors. The differentiation program can be divided into early and late events. Early events are triggered by growth arrest at the G1/S boundary and characterized by the activation of a set of genes (pOb24, lipoprotein lipase, etc.). The expression of the terminal differentiation-related genes takes place after a limited growth resumption of early markers containing cells and requires the presence of permissive hormones (growth hormone and triiodothyronine). Insulin acts solely as a modulator in the expression of the terminal differentiation-related genes. In vivo studies suggest that the acquisition of new adipocytes might result from terminal differentiation of dormant, already commited (pOb24 positive) cells when exposed to appropriate mitogenic or adipogenic stimuli.

adipocyte / differentiation / cell cycle / hormonal regulation 


\section{INTRODUCTION}

L'adipocyte est une cellule hautement différenciée qui joue un rôle central dans la régulation de la balance énergétique chez les espèces supérieures. De manière très schématique, la cellule adipeuse peut être décrite comme un réservoir permettant, d'une part le stockage de l'énergie - sous forme de triglycérides - pendant les périodes fastes, et d'autre part la restitution de cette énergie - sous forme d'acides gras - pendant le jeûne ou l'exercice physique. On considère que chez l'homme normal, plus de $95 \%$ de l'énergie se trouve dans le tissu adipeux. Pour remplir ce rôle physiologique, l'adipocyte est équipé d'une machinerie enzymatique complexe, et présente une sensibilité aiguë à de nombreuses hormones qui contrôlent la balance entre les états de lipogenèse et de lipolyse. Outre ce rôle de réservoir énergétique de l'organisme, le tissu adipeux participe à la régulation d'autres fonctions physiologiques. Ainsi, il a été suggéré que le tissu adipeux produirait des signaux biologiques participant aux contrôles de l'appétit et de la thermogenèse. Par ailleurs, le tissu adipeux jouerait un rôle non négligeable dans la fonction de reproduction.

Le développement excessif de la masse adipeuse est, le plus souvent, lié à une hypertrophie cellulaire, c'est-à-dire à l'augmentation de la taille moyenne des adipocytes due à une accumulation exagérée de triglycérides. Dans les obésités les plus sévères, chez l'animal et chez I'homme, l'hypertrophie s'accompagne d'une augmentation du nombre total de cellules adipeuses. II faut noter que cette hyperplasie peut se déclencher, même à un âge très avancé, chez l'homme et que ce phénomène paraît irréversible puisque chez les obèses la perte de poids consécutive à un régime sévère provient toujours d'une correction de l'hypertrophie mais rarement d'une correction de l'hyper- plasie. Le plus souvent, l'arrêt du régime a pour résultat un retour rapide à l'état d'obésité initial.

La mise en place du tissu adipeux est un phénomène tardif qui se produit selon les espèces soit à la fin de la vie fœtale, soit après la naissance. Le développement de ce tissu se déroule en 2 étapes principales. Le $1^{\text {er }}$ événement est une étape caractérisée par la prolifération de cellules de morphologie fibroblastique appelées préadipocytes. Dans un $2^{\theta}$ temps, ces préadipocytes subissent une différenciation terminale pendant laquelle la cellule se gorge de lipides pour acquérir la morphologie classique de l'adipocyte. De nombreuses observations suggèrent que, même à un âge avancé, des cellules préadipocytaires persistent au sein du tissu adipeux et que, sous l'effet de stimuli non identifiés, ces cellules peuvent se différencier en adipocytes. De plus, le nombre total de cellules adipeuses est soumis à une stricte régulation puisque chez le rat, mais peut-être également chez l'homme, après une lipectomie partielle, un retour au nombre initial d'adipocytes se produit en quelques semaines ou quelques mois. Ces observations permettent de comprendre les nombreux échecs constatés dans les traitements nutritionnels ou chirurgicaux des obésités humaines. L'identification des facteurs impliqués dans la multiplication des préadipocytes et dans leur différenciation terminale paraît indispensable à la compréhension du contrôle de la mise en place normale et pathologique du tissu adipeux.

De nombreuses études, réalisées chez l'homme obèse et dans les divers modèles d'obésités génétiques ou nutritionnelles chez l'animal, ont révélé la grande multiplicité des signaux biologiques impliqués dans les phénomènes d'hyperplasie et d'hypertrophie des cellules adipeuses. Cette complexité évidente a incité certains laboratoires, dont le nôtre, à développer 
des modèles cellulaires appropriés à l'étude des mécanismes de différenciation adipocytaire in vitro.

Depuis une quinzaine d'années, plusieurs lignées cellulaires à potentialité adjpocytaire ont été isolées par différentes techniques. Les lignées préadipocytaires les plus utilisées, 3T3-L1 et 3T3-F442A, ont été isolées par sous-clonage de la lignée 3T3 provenant d'embryons de souris (Green et Kehinde, 1974; 1976). La lignée Ob17 a été obtenue par immortalisation spontanée de cellules du tissu adipeux de souris adultes et génétiquement obèses (Négrel et al, 1978).

\section{LE PROCESSUS DE CONVERSION ADIPOCYTAIRE IN VITRO}

Pendant la phase de prolifération, les cellules de toutes les lignées préadipocytaires présentent une morphologie fibroblastique et n'expriment aucune fonction de différenciation (fig 1A). Après l'arrêt de la prolifération et lorsque les cellules sont maintenues dans un milieu adéquat, se produit la différenciation qui conduit après un temps variable selon la lignée cellulaire (de quelques jours à plusieurs semaines) à l'accumulation intracellulaire de triglycérides (fig $1 \mathrm{C}$-D). Le phénomène de conversion adipocytaire est réservé à certaines cellules de la monocouche cellulaire qui apparaissent regroupées en colonies. L'apparition de ces colonies de cellules adipeuses, phénomène commun à toutes les lignées préadipocytaires, dépend du fait que parmi l'ensemble de la population cellulaire, seules certaines cellules deviennent susceptibles de se différencier alors que d'autres resteront silencieuses, et résulte d'événements mitotiques qui sont spécifiques aux cellules aptes à se différencier. Ces mitoses post-confluentes sont limitées dans le temps, précédent l'accumulation li- pidique (Pairault et Green, 1979; Djian et al, 1982) et conduisent à l'augmentation sélective du nombre de cellules aptes à la différenciation. Pour les cellules 3T3F442A et Ob17, ces mitoses postconfluentes sont critiques pour la différenciation terminale et sont contrôlées par un (ou des) facteur(s) de faible poids moléculaire contenu(s) dans le sérum de veau fœetal (Grimaldi et al, 1982). La fréquence de l'événement qui confère l'état de susceptibilité - ou commitment - dépend à la fois du milieu de culture et de la lignée cellulaire utilisée (Green et Kehinde, 1976; Négrel et al, 1978).

L'expression du programme de différenciation adipocytaire nécessite un environnement hormonal approprié. Pour la plupart des lignées préadipocytaires, un milieu de culture enrichi par $10 \%$ de sérum de veau foetal est propice à la différenciation. Différentes approches expérimentales ont permis l'identification de plusieurs hormones adipogéniques. Ces hormones peuvent être classées en hormones permissives, dont la présence dans le milieu de culture est indispensable à la différenciation, et en hormones modulatrices qui affectent l'amplitude de l'accumulation lipidique. Ainsi, l'hormone somatotrope (GH) (Morikawa et al, 1982; Doglio et al, 1986) et les hormones thyroïdiennes (Grimaldi et al, 1982) exercent un effet permissif sur la différenciation des cellules 3T3-F442A et Ob17 alors que l'insuline (Grimaldi et al, 1983; Amri et al, 1984) et les glucocorticoïdes (Chapman et al, 1985) sont des modulateurs de la conversion adipocytaire.

Les changements morphologiques des cellules lors de la différenciation s'accompagnent d'un profond bouleversement au niveau biochimique. La conversion adipocytaire conduit à la modification des contenus cellulaires de plus d'une centaine de protéines (Shidu, 1979) et à l'apparition de nombreuses protéines non exprimées avant différenciation (Spiegelman et 
$\mathbf{A}$

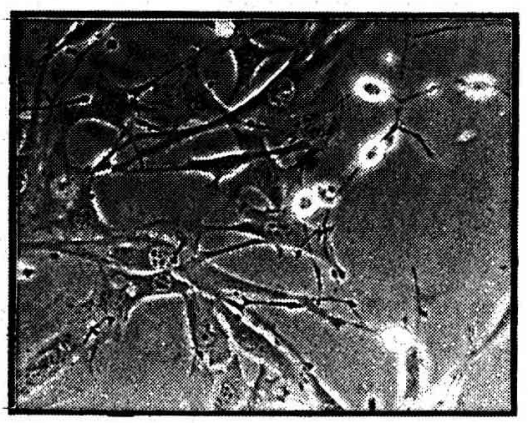

C

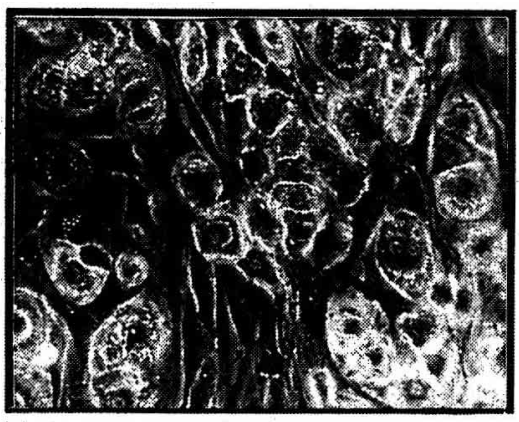

B

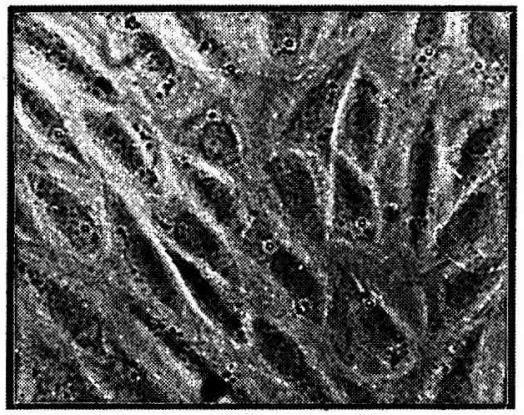

D

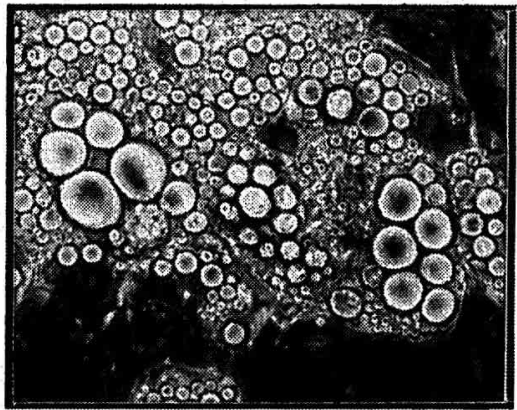

Flg 1. Changements morphologiques des cellules Ob17 au cours de la différenciation adipocytaire. Les cellules cultivées dans un milieu contenant $10 \%$ de sérum de veau foetal ont été photographiées en phase de croissance $(A)$, à confluence $(B)$ et aux jours $8(C)$ et $15(D)$ de la phase post-confluente $(x 400)$.

Green, 1980). Beaucoup de ces protéines sont des enzymes impliquées dans le métabolisme des acides gras et des triglycérides comme l'acétyl CoA carboxylase, l'acide gras synthétase, l'enzyme malique, la glycérophosphate déshydrogénase (GPDH), la lipase hormonosensible ou la lipoprotéine lipase (LPL). Outre l'induction de ces protéines déjà connues, différents travaux ont permis la mise en évidence de marqueurs de différenciation dont les fonctions ne sont pas encore identifiées. Parmi ces nouveaux marqueurs, on peut citer : la protéine $\mathrm{aP}_{2}$ qui présente une forte homologie avec les transporteurs des acides gras (Bernlohr et al, 1984; Cook et al, 1985b), l'adipsine (Adn) qui est une protéine sécrétée par l'adipocyte et qui présente de fortes homologies avec les protéases à sérine (Cook et al, 1985a) et les ARN messagers pOb24 (Amri et al, 1986b) et clone 5 (Navre et Ringold, 1988) qui codent pour des protéines encore inconnues. Dans pratiquement tous les cas étudiés, l'induction des marqueurs protéiques de différenciation s'accompagne de l'augmentation des ARNm correspondants. Le plus souvent ces variations des contenus en ARNm proviennent de l'activation transcriptionnelle des gènes concernés 
(Cook et al, 1985b; Bernlohr et al, 1985; Dani et al, 1986).

\section{RÉGULATION DE LA DIFFÉRENCIATION ADIPOCYTAIRE}

Les travaux effectués ces dernières années ont révélé la grande complexité des mécanismes impliqués dans l'activation et dans la modulation de l'expression des gènes de différenciation adipocytaire. Même si, à l'heure actuelle, ces mécanismes régulationnels restent encore mal connus, on peut toutefois affirmer que l'expression du programme de différenciation adipocytaire est soumise à des contrôles multiples et que ces contrôles peuvent être différents selon le gène considéré.

\section{Événements précoces et tardifs de la conversion adipocytaire}

In vitro, le processus de différenciation adjpocytaire comporte clairement des événements précoces et des événements tardifs. Cette distinction a été établie d'une part sur des bases cinétiques et d'autre part en raison de la régulation différentielle de l'expression des gènes précoces et tardifs de conversion adipocytaire.

L'étude des cinétiques d'apparition des marqueurs de différenciation des cellules Ob17 révèle que certains d'entre eux, comme l'activité LPL, sont induits très tôt et atteignent leur niveau maximal d'expression avant l'accumulation de lipides. Pour d'autres, comme l'activité GPDH ou pour les activités de synthèse des acides gras, l'induction est plus tardive et coïncide avec la différenciation morphologique des cellules (Vannier et al, 1985). Lors de la différenciation des cellules 3T3-F442A, l'accumulation des ARNm GPDH et $\mathrm{aP}_{2}$ est antérieure à celle de l'ARNm adipsine
(Cook et al, 1985a). Ces arguments cinétiques ne peuvent toutefois à eux seuls constituer une preuve définitive de l'absence de coordination de l'induction des marqueurs de différenciation puisque leurs niveaux d'expression sont très variables. Ainsi, dans une cellule pleinement différenciée, le contenu en LPL est 50 a 100 fois plus faible que le contenu en protéine $\mathrm{aP}_{2}$.

Des preuves beaucoup plus convaincantes de l'existence de sous-programmes distincts dans le processus de différenciation adipocytaire sont apportées par la mise en évidence de la régulation différentielle de leur expression. L'obtention du variant cellulaire Ob1754, sous-clone de la lignée Ob17 (Amri et al, 1986c), et l'isolement de la sonde pOb24 complémentaire à un ARNm spécifique de la phase précoce de la conversion adipocytaire nous ont récemment permis de contirmer l'existence de sous-programmes d'expressions précoce et tardive.

Les cellules Ob1754, contrairement aux cellules des autres lignées préadypocytaires, sont incapables d'accumuler des lipides lorsqu'elles sont maintenues dans un milieu de culture enrichi par $10 \%$ de sérum de veau foetal (fig 2a). Le traitement chronique par un mélange de méthylglyoxal bis (guanylhydrazone) (MGBG) et de putrescine restaure la différenciation des cellules Ob1754 (fig 2b). Des études complémentaires ont montré que ces cellules Ob1754 ont un métabolisme anormal des polyamines et qu'elles ne peuvent accumuler, pendant la phase de confluence, la spermidine; le traitement de ces cellules par le mélange MGBG/putrescine rétablit une augmentation de la concentration en cette polyamine, événement indispensable à la conversion adipocytaire (Amri et al, 1986a, b).

La détermination des activités spécifiques de la LPL et de la GPDH au cours de la phase post-confluente montre que pour les cellules Ob1754, l'induction de l'activité 

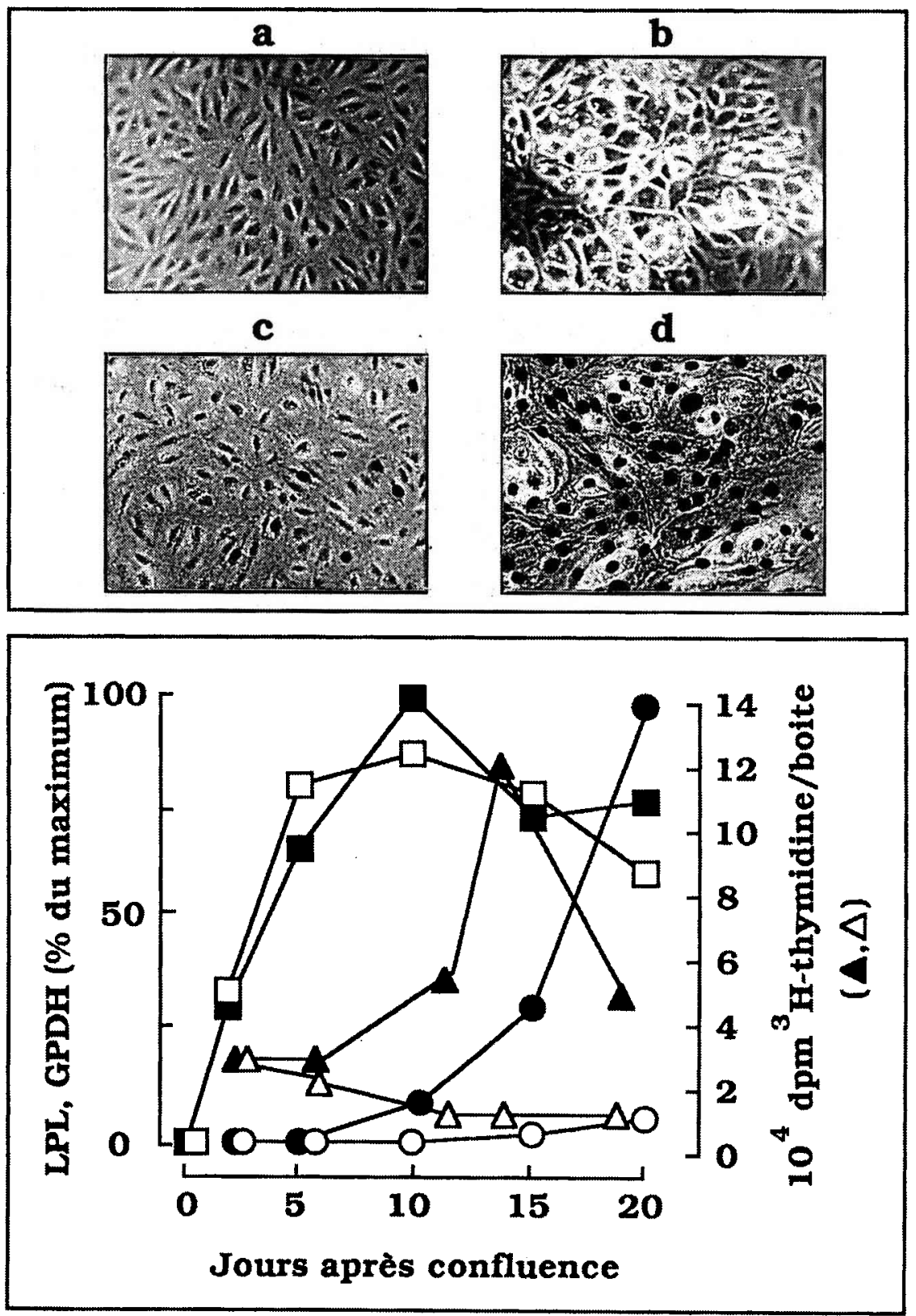

Fig 2. Effets du traitement par le MGBG et la putrescine sur la différenciation des cellules Ob1754. Les cellules Ob1754 ont été maintenues depuis la confluence dans un milieu contenant $10 \%$ de

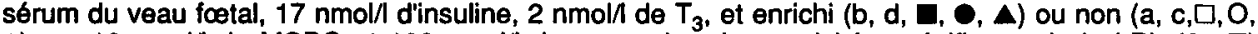
$\Delta)$ par $10 \mu \mathrm{mol} / /$ de MCBG et $100 \mu \mathrm{mol} / /$ de putrescine. Les activités spécifiques de la LPL $(\square, \square)$ GPDH $(O, \theta)$ et le taux d'incorporation de thymidine tritiée dans l'ADN $(\Delta, \Delta)$ ont été déterminés aux temps indiqués. Les photographies a et b ont été réalisées au jour 18 après confluence. Les photographies $c$ et $d$ représentent des autoradiographies après une exposition des cellules pendant $48 \mathrm{~h}$ (jours 11 à 13) à la thymidine tritiée $(\times 300)$. 
GPDH nécessite l'augmentation de la concentration intracellulaire en spermidine induite par le traitement MGBG/putrescine alors que l'induction de l'activité LPL se produit au même niveau et avec une cinétique identique dans les cellules traitées ou non (fig 2). Comme l'activité GPDH, le déroulement des mitoses post-confluentes est dépendant du traitement des cellules par le mélange MGBG/putrescine (fig $2 \mathrm{c}$, d). Ces mitoses post-confluentes sont postérieures à l'induction de la LPL et précèdent celle de l'activité GPDH (fig 2). Cette séquence d'événements - induction de la LPL, mitoses post-confluentes, induction de la GPDH - a été démontrée pour d'autres modèles de différenciation adipocytaire, dont la lignée parentale Ob17 et les cellules 3T3-F442A (Amri et al, 1986a).

Ces résultats obtenus en culture cellulaire sont à rapprocher d'observations in vivo lors de la mise en place du tissu adipeux chez le rongeur qui montraient que, d'une part, la lipoprotéine lipase peut être détectée avant toute accumulation lipidique dans les cellules préadipocytaires de la région inguinale chez le rat nouveau-né (Péquignot-Planche et al, 1977) et que d'autre part chez la souris, l'apparition de l'activité GPDH et de l'accumulation lipidique se produit dans des cellules qui subissent au préalable des événements mitotiques (Cook et Kozak, 1982).

\section{Clonage d'un ADN complémentaire d'un ARNm marqueur de l'état préadipocytaire}

Le criblage différentiel de banques d'ADNc est une technique très performante pour l'isolement de sondes ADN complémentaires des ARNm spécifiquement induits pendant un phénomène de différenciation. Cette méthode a permis l'isolement de plusieurs ADN complémentaires des mar- queurs de la différenciation adipocytaire comme l'adipsine (Spiegelman et al, 1983), la protéine $\mathrm{aP}_{2}$ (Bernlohr et al, 1984) ou des marqueurs non encore identifiés (Chapman et al, 1984). Dans notre laboratoire, nous nous sommes plus spécifiquement intéressés au clonage d'ADN complémentaire des messagers induits à un niveau important dès le début de la phase de confluence. Dans ce but, une banque d'ADN complémentaire aux ARNm provenant de cellules Ob17 peu différenciées (jour 4 après confluence) a été soumise à un criblage par des ADNc préparés à partir, soit de cellules non différenciées (cellules en prolifération), soit de cellules différenciées. La sonde pOb24, obtenue par cette technique, détecte un ARNm de $6 \mathrm{~kb}$ dans les cellules Ob17 et 3T3-F442A en cours de différenciation. Cet ARNm pOb24 est absent de ces cellules en prolifération et des cellules dépourvues de potentialité adipocytaire avant ou après confluence. L'analyse de divers tissus de souris adulte confirme la spécificité adipocytaire de l'expression de l'ARNm pOb24 puisque cet ARN n'est retrouvé que dans les tissu adipeux blanc et brun, et est absent de tous les tissus testés dont le foie, le muscle, le rein ou le cerveau (Dani et al, 1989b).

Pendant la conversion adipocytaire des cellules Ob17 et 3T3-F442A l'accumulation de l'ARNm pOb24 est très rapide et précède celles des ARNm GPDH et adipsine (fig $3 A$ et B). De plus, l'expression de l'ARNm pOb24 diminue dans les 2 lignées cellulaires en fin de différenciation.

Cette observation, qui suggère qu'en culture l'expression de l'ARNm pOb24 est maximale dans la phase précoce de différenciation, a été confirmée in vivo. En effet, comme le montre la figure $3 \mathrm{C}$, le signal pOb24 observé dans le tissu adipeux total est majoritairement apporté par les cellules de la fraction stromale du tissu qui contient les préadipocytes, alors que la 
fraction adipocytaire qui contient les cellules pleinement différenciées est très pauvre en messager pOb24. La situation inverse est observée entre les 2 fractions du tissu pour les ARNm GPDH et adipsine qui sont concentrés dans les adipocytes. La mesure du taux de transcription du gène pOb24 montre que ces variations des contenus en ARNm durant la différenciation des cellules Ob17, ou dans les fractions stromale et adipocytaire du tissu adipeux, sont reliées à des variations de l'activité transcriptionnelle.

II est intéressant de noter que cette distribution inverse dans les fractions stromale et adipocytaire pour l'ARNm pOb24 et les ARNm GPDH et adipsine est retrouvée dans tous les sites adipeux étudiés (périrénal, périovarien, périépididymaire, inguinal), aussi bien pour des animaux jeunes que pour des animaux âgés (4 à 24 semaines).

Ces différentes observations démontrent que l'expression de l'ARNm pOb24 peut être considérée, au même titre que celle de la $\mathrm{LPL}$, comme un événement précoce du processus de différenciation des cellules en culture. Une différence dans l'expression de ces 2 gènes peut cependant être notée puisque, au contraire du gène pOb24, l'expression du gène LPL ne diminue pas dans les cellules pleinement différenciées en culture et ne disparaît pas dans les adipocytes mûrs du tissu adipeux.

\section{Régulation différentielle de l'expression des gènes précoces et tardifs de différenciation adipocytaire}

Comme cela a déjà été signalé, l'expression de la différenciation terminale nécessite des événements mitotiques qui surviennent après confluence et qui sont réservés à certaines cellules dites "engagées". L'utilisation des cellules Ob1754 pour lesquelles ces mitoses ne se produisent que dans un milieu de culture supplémenté en MGBG/putrescine permet de montrer que l'activation de la transcription des gènes pOb24 et LPL se produit dans les cellules confluentes en l'absence de tout traitement alors que l'activation de gènes d'expression tardive, comme ceux de la GPDH ou de la protéine $\mathrm{aP}_{2}$, nécessite absolument une augmentation intracellulaire en spermidine et les événements mitotiques de la phase de confluence (Amri et al, 1986b).

L'étude du mode d'action de l'insuline, de l'hormone somatotrope (GH) et de la triiodothyronine $\left(T_{3}\right)$ sur l'expression des gènes de différenciation a montré que les effets permissifs et modulateurs de ces hormones ne s'exercent en fait que sur l'expression du programme de différenciation terminale, alors que l'expression des gènes précoces comme pOb24 et LPL est indépendante de ce contrôle multihormonal (Doglio et al, 1986; Dani et al, 1989b). Cette différence dans le contrôle multihormonal des gènes précoces et tardifs est illustrée dans la figure 4 pour des cellules Ob17 exposées ou non pendant la phase de confluence à des concentrations physiologiques en insuline, en $\mathrm{T}_{3}$ ou en $\mathrm{GH}$. L'accumulation de l'ARNm GPDH nécessite le traitement des cellules par les 3 hormones alors que l'expression des ARNm pOb24 et LPL se produit de manière similaire dans les cellules, exposées ou non aux 3 hormones. En ce qui concerne les effets de la $\mathrm{GH}$, ces résultats ont été corroborés au niveau transcriptionnel puisque le traitement par l'hormone est nécessaire à l'activation du gène d'expression tardive $\mathrm{aP}_{2}$, alors que le taux de transcription du gène pOb24 est important dès la confluence dans les cellules exposées ou non à la GH (Doglio et al, 1986). 


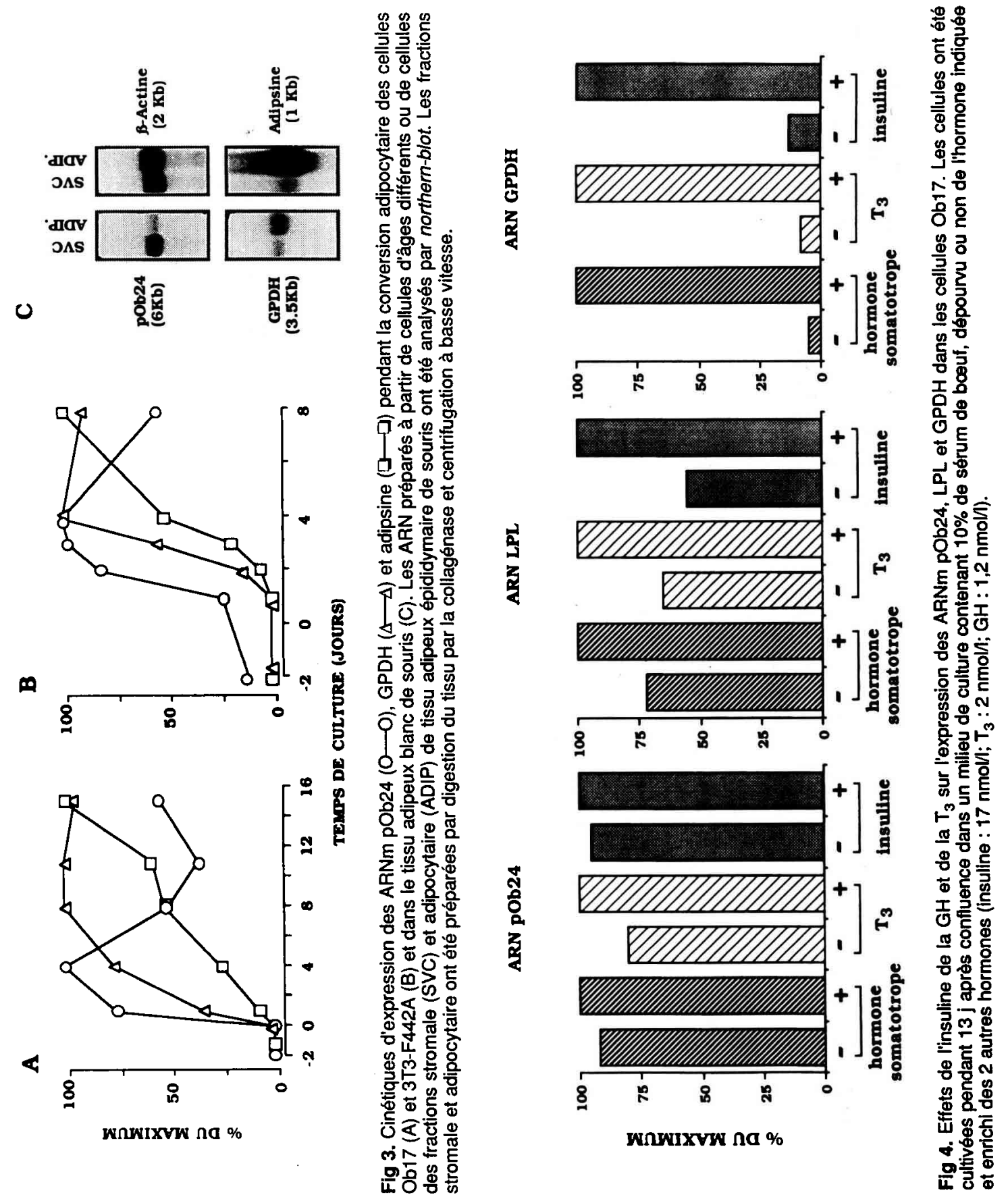


Régulation de l'expression des gènes précoces de conversion adipocytaire

Plusieurs preuves expérimentales suggèrent que l'engagement des préadipocytes dans la différenciation est directement relié à l'arrêt de la prolifération cellulaire. Une telle relation entre l'arrêt de la prolifération cellulaire et la différenciation a été proposée pour la différenciation des cellules sanguines (Lajtha et al, 1978) ou celle des cellules musculaires (NadalGinard, 1978). Dans ces 2 exemples, un arrêt des cellules précurseurs dans la phase G1 du cycle cellulaire paraît indispensable au commitment.

Pour les préadipocytes $3 T 3-L 1$, un état d'arrêt des cellules dans un état précis de la phase $\mathrm{G1}_{1}$, état appelé $\mathrm{GD}$, a été décrit comme étant nécessaire à la différenciation (Wille et Scott, 1983). Ces études, bien que très intéressantes, sont toutefois à considérer avec prudence puisque fondées sur l'étude de la différenciation terminale et réalisées avec des cellules déjà confluentes. Dans notre laboratoire, une étude portant sur l'expression de 2 gènes marqueurs des événements très précoces de la différenciation, les gènes pOb24 et LPL, réalisée par synchronisation des cellules Ob17 et 3T3-F442A avant confluence, permet d'affirmer que le commitment des préadipocytes nécessite l'arrêt prolongé des cellules dans la phase G1 tardive du cycle cellulaire (Amri et al, 1986b; Dani et al, 1989b).

L'arrêt de la prolifération des cellules Ob17 et 3T3-F442A peut être aisément obtenu avant confluence, soit par addition d'aphidicholine (inhibiteur de l'ADN polymérase) ou de thymidine à forte concentration, soit par appauvrissement du milieu de culture en calcium ou en sérum. Dans tous les cas, l'arrêt de la prolifération cellulaire est rapide, totalement réversible et se produit en G1 tardif. L'analyse des ARN des cellules soumises pendant $24 \mathrm{~h}$ à ces traitements révèle une forte expression des ARNm pOb24 et LPL. Le retour à l'état prolifératif, par retrait de l'inhibiteur, provoque une disparition de l'expression des 2 ARNm. Ces phénomènes d'induction de l'ARNm pOb24 après blocage de la prolifération, et de disparition après reprise de la prolifération cellulaire, sont directement correlés avec l'activation et l'inactivation transcriptionnelles du gène correspondant comme le montrent les résultats présentés dans la figure 5 . Dans cette expérience, le gène de la dihydrofolate réductase (DHFR) a été choisi comme marqueur de la synchronisation des cellules en G1 tardif, puisqu' il a été rapporté que l'expression de ce gène est maximale pendant cette phase et faible pendant les autres phases du cycle cellulaire (Farnham et Schimke, 1985). Cette expérience démontre donc que l'arrêt des cellules Ob17 provoque l'activation du gène pOb24 et l'augmentation de la transcription du gène DHFR. Les taux de transcription des 2 gènes sont très rapidement diminués dès que les cellules entament la phase $S$. Cette perte d'induction des gènes pOb24 et DHFR ne nécessite pas de synthèse protéique, ce qui suggère que la transcription de ces gènes serait sous contrôle d'un facteur actif pendant la phase G1 et très rapidement inactivé au début de la phase $S$ (Dani et al, 1989b).

Ces résultats suggèrent fortement que l'expression du programme précoce de différenciation et donc le commitment des préadipocytes sont reliés à l'arrêt des cellules précurseurs dans un état précis de la phase G1 du cycle cellulaire. On peut supposer que, lorsque les cellules arrivent à confluence, seules certaines d'entre elles se trouvent dans cet état compatible avec la différenciation alors que les autres cellules, qui sont dans un état différent, restent silencieuses. Cette distinction déterminée à confluence ne semble pas irré- 


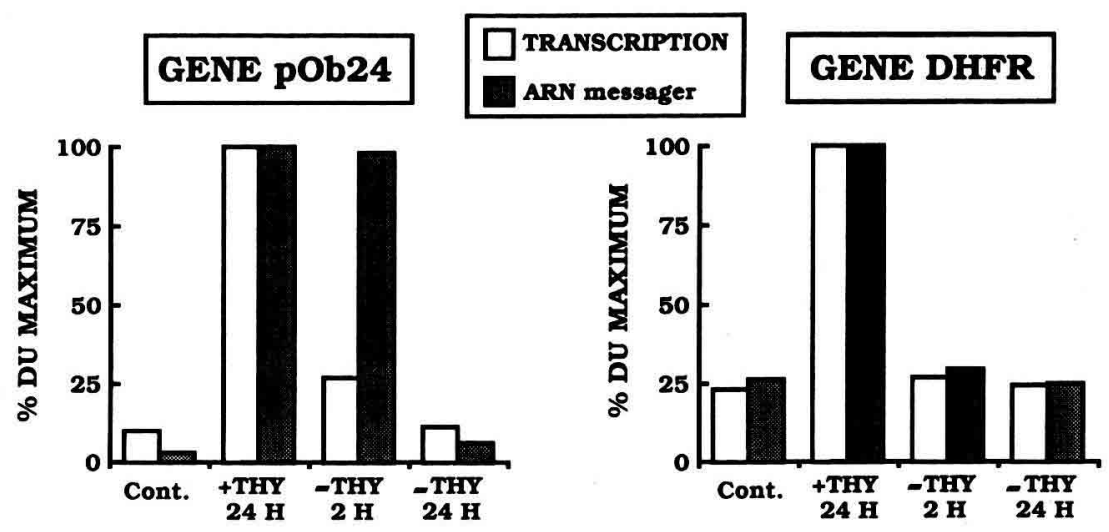

Fig 5. Expression des gènes pOb24 et DHFR en fonction du cycle cellulaire dans les cellules Ob17. Les cellules en phase de croissance exponentielle (Cont.) ont été traitées pendant $24 \mathrm{~h}$ par $5 \mathrm{mmol} / \mathrm{l}$ de thymidine (+ THY $24 \mathrm{H}$ ). L'arrêt du blocage par la thymidine (-THY) a été réalisé par lavage des cellules et addition de $10 \mu \mathrm{mol} / \mathrm{l}$ de cytidine. Aux temps indiqués les contenus en ARNm ont été déterminés par northern-blot et les taux de transcription des gènes ont été estimés par la technique d'élongation des chaînes naissantes d'ARN sur noyaux isolés.

médiable. La transition vers l'état d'aptitude pour une cellule silencieuse reste possible même très tard après confluence à condition que la cellule ait la possibilité de proliférer à nouveau. Une telle transition vers l'état d'aptitude est rendue possible, par exemple, lorsqu' une plaie est créée mécaniquement dans une zone non différenciée de la monocouche cellulaire (Djian et al, 1982). De même, la perte d'aptitude à la différenciation est également possible (Ignots et Massagué, 1985; Cornelius et al, 1988). Ainsi, au début de la confluence, le traitement de cellules préadipocytaires par des mitogènes puissants tels le FGF (fibroblast growth factor) ou la prostaglandine $F 2 \alpha$ empêche la différenciation; après la prolifération active induite par ces facteurs de croissance, la probabilité d'arrêt des cellules dans la phase compatible avec le commitment deviendrait très faible, voire nulle (fig 6).

\section{Régulation de l'expression des gènes de differenciation terminale}

Les données récentes suggèrent que les mécanismes de l'activation et de la modulation de l'expression des gènes de la différenciation terminale ne seraient pas communs à l'ensemble du sous-programme mais, au contraire, que chacun des gènes serait soumis à des contrôles particuliers.

Concernant la modulation de l'expression de la différenciation terminale, des différences fondamentales ont été mises en évidence pour le mode d'action de l'insuline sur l'expression des gènes GPDH, phosphoénol pyruvate carboxykinase (PEPCK) et Adn. En effet dans les cellules Ob17 et 3T3-F442A, l'insuline exerce des effets stimulateurs sur le niveau d'accumulation des ARNm GPDH et PEPCK et un puissant effet négatif sur le niveau de 


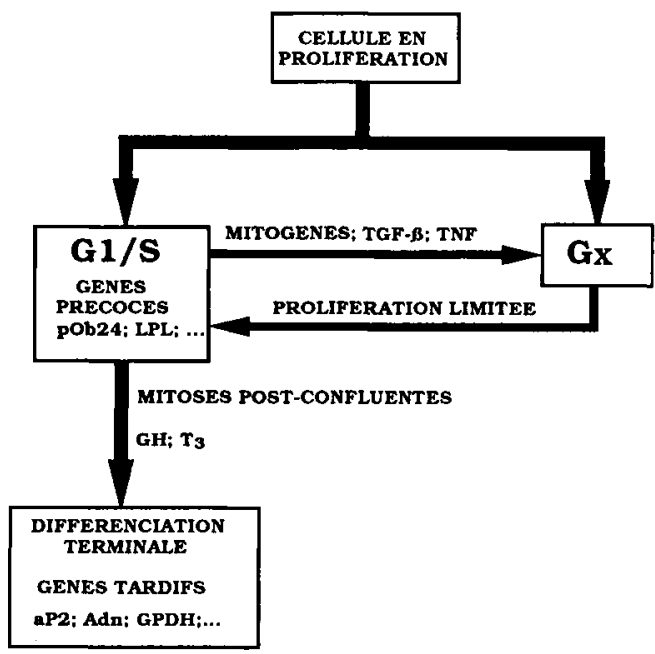

Fig 6. Les étapes de la différenciaton adipocytaire in vitro. (TGFB: Transforming Growth Factor $\beta$; TNF: Tumor Necrosis Factor).

l'ARNm Adn. De plus, les niveaux de régulation de l'expression de ces 3 gènes par l'hormone sont différents : les effets inhibiteurs sur l'expression du gène Adn sont purement transcriptionnels alors que les effets stimulateurs sur les gènes GPDH et PEPCK sont, dans le fer cas en partie transcriptionnels, et uniquement posttranscriptionnels dans le $2^{\circ}$ cas (Dani et al, 1986; Dani et al, 1989a).

Les mécanismes impliqués dans l'activation des gènes de différenciation terminale paraissent également différents selon le gène considéré. Cela peut, en particulier, être observé dans les cellules Ob1754 pour l'activation des gènes $\mathrm{aP}_{2}$, $\mathrm{Adn}$ et GPDH, en relation avec les $\mathrm{mi}$ toses post-confluentes et l'hormone somatotrope. La figure 7 présente les résultats d'une expérience dans laquelle les cellules Ob1754 maintenues après confluence dans un milieu de culture contenant ou non de la $\mathrm{GH}$ ont été traitées par le mélange MGBG/putrescine, in- ducteur des mitoses post-confluentes. L'analyse par northern-blot des ARN de ces cellules montre, comme attendu, que l'accumulation de l'ARNm GPDH, rendant compte de l'activité transcriptionnelle du gène, nécessite à la fois un traitement à long terme par la GH (Doglio et al, 1986) et des mitoses post-confluentes (Pairault et Green, 1979; Djian et al, 1982). Si l'accumulation de l'ARNm Adn nécessite également l'exposition des cellules à la $\mathbf{G H}$, elle est au contraire tout à fait indépendante de l'augmentation de la concentration intracellulaire en spermidine et des mitoses post-confluentes induites par le traitement MGBG/putrescine. Enfin, l'induction du gène $\mathrm{aP}_{2}$ paraît strictement dépendante du traitement MGBG/putrescine et ne pas nécessiter la GH.

Ces observations, confirmées pour les cellules de la lignée parentale Ob17, illustrent le dégré de complexité des mécanismes impliqués dans l'activation des gènes de différenciation terminale. La complexité de ces mécanismes est, sans aucun doute, encore plus grande si l'on tient compte des autres hormones (dont la $T_{3}$ ) et des autres facteurs encore non identifiés qui exercent un rôle permissif sur l'expression de la différenciation terminale des adipocytes.

\section{CONCLUSIONS}

In vitro, le processus de conversion adipocytaire comporte clairement des événements précoces et tardifs. Le démarrage du programme de différenciation paraît uniquement lié à l'arrêt des cellules dans la phase G1 du cycle cellulaire et est caractérisé par l'expression de plusieurs gènes, notamment lipoprotéine lipase et pOb24. Ces cellules «pré-différenciées" engagent ensuite leur programme de différenciation terminale caractérisé par l'ac- 

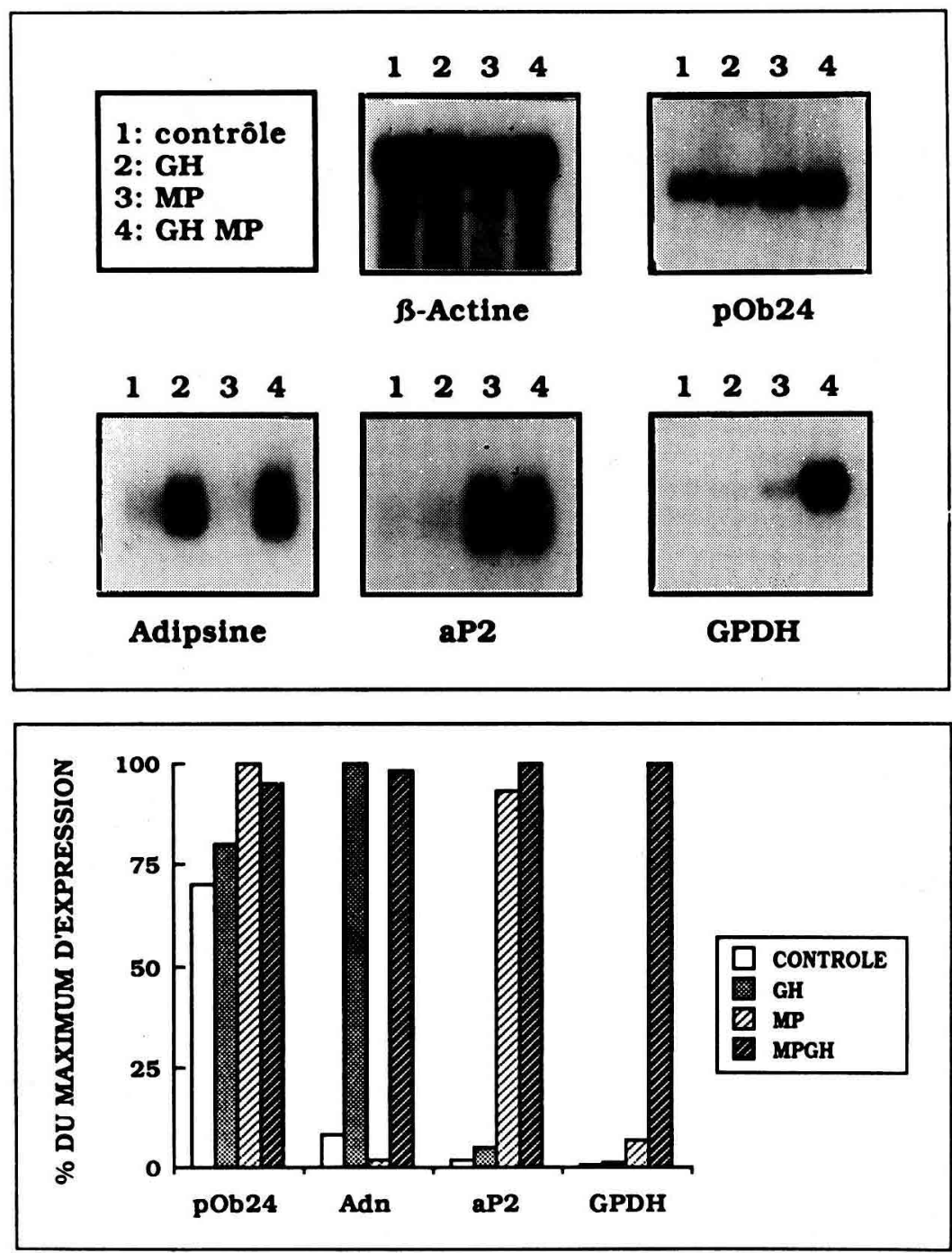

Fig 7. Contrôle de l'expression des gènes de différenciation terminale par la GH et par le mélange MGBG/putrescine dans les cellules Ob1754. Les cellules ont été maintenues pendant $15 \mathrm{j}$ après confluence dans un milieu contenant $10 \%$ de sérum de bœuf, $17 \mathrm{nmol} / /$ d'insuline, $2 \mathrm{nmol} / / \mathrm{de} \mathrm{T}_{3}$, enrichi ou non par $1,2 \mathrm{nmol} /$ de $\mathrm{GH}$ et en l'absence ou la présence de MGBG $(10 \mu \mathrm{mol} / \mathrm{l})$ et de putrescine $(100 \mu \mathrm{mol} / \mathrm{)})$ (mélange MP). Les ARNm ont été quantifiés par northern-blot.

quisition de la capacité de synthétiser et d'utiliser les triglycérides. L'expression des divers gènes de cette différenciation termi- nale est contrôlée par plusieurs hormones et nécessite des événements mitotiques préalables (fig 6). 
L'extrapolation de ce modèle, établi in vitro, au développement in vivo du tissu adipeux chez l'homme ou chez l'animal est encore prématurée. Toutefois la mise en évidence, d'une part de l'existence au sein du tissu adipeux de cellules dépourvues de lipides et de marqueurs tardifs mais exprimant les marqueurs précoces pOb24 et. LPL et, d'autre part, de l'existence, pour les préadipocytes, d'événements mitotiques qui précèdent l'acquisition de la GPDH et des lipides, suggère que dans ces grandes lignes un tel modèle pourrait rendre compte des événements de différenciation des adipocytes in vivo. Les travaux engagés, en particulier par des techniques d'hybridation in situ, devraient permettre, dans un futur relativement proche, une meilleure connaissance des phases de prolifération et de différenciation terminale des préadipocytes et donc du développement normal et pathologique du tissu adipeux.

\section{RÉFÉRENCES}

Amri E, Grimaldi P, Négrel R, Ailhaud G (1984) Adipose conversion of Ob17 cells: insulin acts solely as a modulator in the expression of the differentiation program. Exp Cell Res 152, 368-377

Amri E, Barbaras R, Doglio A, Dani C, Grimaldi $P$, Ailhaud G (1986a) Role of spermidine in the expression of late markers of adipose conversion. Effects of growth hormone. Biochem J 239, 363-370

Amri E, Dani C, Doglio A, Grimaldi P, Ailhaud G (1986b) Coupling of growth arrest and expression of early markers during adipose conversion of preadipocyte cell lines. Biochem Biophys Res Commun 137, 903-910

Amri E, Dani C, Doglio A, Etienne J, Grimaldi P, Ailhaud G (1986c) Adipose cell differentiation: evidence for a two-step process in the polyamine-dependent Ob1754 clonal line. Biochem J 238, 115-122

Bernlhor DA, Angus CW, Lane MD, Bolanowski MA, Kelly TJ (1984) Expression of specific
mRNAs during adipose differentiation: identification of an mRNA encoding a homologue of myelin $\mathrm{P}_{2}$ protein. Proc Natl Acad Sci USA 81, 5468-5472

Bernlohr DA, Bolanowski MA, Kelly TJ, Lane MD (1985) Evidence for an increase in transcription of specific mRNAs during differentiation of 3T3-L1 preadipocytes. J Biol Chem 260, 5563-5567

Chapman AB, Knight DM, Dieckmann BS, Ringold GM (1984) Analysis of gene expression during differentiation of adipogenic cells in culture and hormonal control of the developmental program. J Biol Chem 259, 1554815555

Chapman AB, Knight DM, Ringold GM (1985) Glucocorticoid regulation of adipocyte differentiation: hormonal triggering of the developmental program and induction of a differentiation-dependent gene. J Cell Biol 101, 1227-1235

Cook JR, Kozak LP (1982) sn-glycero-3phosphate dehydrogenase gene expression during mouse adipocyte development in vivo. Dev Biol 92, 440-448

Cook KS, Groves DL, Min HY, Spiegelman BM (1985a) A developmentally regulated mRNA from 3 T 3 adipocytes encodes a novel serine protease homologue. Proc Natl Acad Sci USA 82, 6480-6484

Cook KS, Hunt CR, Spiegelman BM (1985b) Developmentally regulated mRNAs in 3T3 adipocytes: analysis of transcriptional control. J Cell Biol 100, 514-520

Cornelius P, Enerback S, Bjursell G, Olivecrona T, Pekala PH (1988) Regulation of lipoprotein lipase mRNA in 3T3-L1 cells by tumour necrosis factor. Biochem J 249, 765-769

Dani C, Doglio A, Grimaldi P, Ailhaud G (1986) Expression of the phosphoenolpyruvate carboxykinase gene and its insulin regulation during differentiation of preadipose cell lines. Biochem Biophys Res Commun 138, 468475

Dani C, Bertrand B, Bardon S, Doglio A, Amri E, Grimaldi P (1989a) Régulation of gene expression by insulin in adipose cells: opposite effects on adipsin and glycerophosphate dehydrogenase genes. Mol Cell Endocrinol 63, 199-208

Dani C, Doglio A, Amri E, Bardon S, Fort P, Bertrand B, Grimaldi P, Ailhaud G (1989b) Cloning and regulation of a mRNA specifical- 
Iy expressed in the preadipose state. $J$ Biol Chem 264, 10119-10125

Djian P, Grimaldi P, Négrel R, Ailhaud G (1982) Adipose conversion of $\mathrm{Ob} 17$ preadipocytes: relationships between cell division and fat cell cluster formation. Exp Cell Res 142, 273281

Doglio A, Dani C, Grimaldi P, Ailhaud G (1986) Growth hormone regulation of the expression of differentiation-dependent genes in preadipocyte Ob1771 cells. Biochem J 238, 123129

Farnham PJ, Schimke RT (1985) Transcriptional regulation of mouse dihydrofolate reductase in cell cycle. J Biol Chem 260, 7675-7680

Green $H$, Kehinde $O$ (1974) Sublines of mouse 3T3 cells that accumulate lipids. Cell 1, 113116

Green H, Kehinde O (1976) Spontaneous heritable changes leading to increased adipose conversion in 3T3 cells. Cell 7, 105-113

Grimaldi P, Djian P, Négrel R, Ailhaud G (1982) Differentiation of $\mathrm{Ob} 17$ preadipocytes to adipocytes: requirement of adipose conversion factor(s) for fat cell cluster formation. Embo $J$ 1, 687-692

Grimaldi P, Djian P, Forest C, Poli P, Négrel $R$, Ailhaud G (1983) Lipogenic and mitogenic effects of insulin during conversion of Ob17 cells to adipose-like cells. Mol Cell Endocrinol 29, 271-285

Ignotz R, Massagué J (1985) Type $\beta$ transforming growth factor controls the adipogenic differentiation of 3 T3 fibroblasts. Proc Natl Acad Sci USA 82, 8530-8534

Lajtha LG, Lord BI, Dexter TM, Wright EG, Allen TA (1978) Interrelationship of differentiation and proliferation control in hematopoietic stem cells. In: Cell differentiation and neoplasia (GF Saunders, ed), Raven Press, New York, 179-209

Morikawa M, Nixon T, Green H (1982) Growth hormone and the adipose conversion of 3T3 cells. Cell 29, 783-789
Nadal-Ginard B (1978) Commitment, fusion and biochemical differentiation of myogenic cell line in the absence of DNA synthesis. Cell $15,855-864$

Navre M, Ringold GM (1988) A growth factorrepressible gene associated with protein kinase C-mediated inhibition of adipocyte differentiation. J Cell Biol 107, 279-286

Négrel R, Grimaldi P, Ailhaud G (1978) Etablishment of preadipocyte clonal line from epididymal fat pad of ob/ob mouse that responds to insulin and to lipolytic hormones. Proc Natl Acad Sci USA 75, 6054-6058

Pairault J, Green H (1979) A study of the adipose conversion of suspended 3 T 3 cells by using glycerophosphate dehydrogenase as differentiation marker. Proc Natl Acad Sci USA 76, 5138-5142

Péquignot-Planche $E$, De Gasquet $P$, Boulangé A, Tonnu NT (1977) Lipoprotein lipase activity at onset of development of white adipose tissue in newborn rats. Biochem $J 162,462$ 463

Shidu R (1979) Two-dimentional electrophoretic analyses of proteins synthetized during differentiation of 3T3-L1 preadipocytes. J Biol Chem 254, 11111-11118

Spiegelman B, Green H (1980) Control of specific protein biosynthesis during the adipose conversion of 3 T3 cells. J Biol Chem 255, 8811-8818

Spiegelman BM, Franck M, Green H (1983) Molecular cloning of mRNA from 3 T3 adipocytes. J Biol Chem 258, 10083-10089

Vannier C, Gaillard D, Grimaldi P, Amri E, Djian $P$, Cermolacce C, Forest C, Etienne J, Négrel R, Aishaud G (1985) Adipose conversion of Ob17 cells and hormone-related events. Int J Obes 9, 41-53

Wille JJ, Scott RE (1983) Topography of the predifferentiation GD growth arrest state relative to other growth arrest states in the G1 phase of the cell cycle. J Cell Physiol 112, 115-122 